\title{
Analyse et évaluation de l'argumentation dans l'analyse critique du discours (CDA) : délibération et dialectique des Lumières
}

Analysis and Evaluation of Argumentation in Critical Discourse Analysis:

Deliberation and the Dialectic of Enlightenment

Isabela Fairclough et Norman Fairclough

Traducteur : Sivan Cohen-Wiesenfeld

\section{OpenEdition}

Journals

Édition électronique

URL : http://journals.openedition.org/aad/1369

DOI : $10.4000 /$ aad. 1369

ISSN : 1565-8961

Éditeur

Université de Tel-Aviv

Référence électronique

Isabela Fairclough et Norman Fairclough, « Analyse et évaluation de l'argumentation dans l'analyse critique du discours (CDA) : délibération et dialectique des Lumières », Argumentation et Analyse du Discours [En ligne], 9 | 2012, mis en ligne le 15 octobre 2012, consulté le 23 septembre 2019. URL: http://journals.openedition.org/aad/1369; DOI : 10.4000/aad.1369

Ce document a été généré automatiquement le 23 septembre 2019.

\section{(†)

Argumentation \& analyse du discours est mis à disposition selon les termes de la licence Creative Commons Attribution - Pas d'Utilisation Commerciale - Pas de Modification 4.0 International. 


\title{
Analyse et évaluation de l'argumentation dans l'analyse critique du discours (CDA) : délibération et dialectique des Lumières
}

\author{
Analysis and Evaluation of Argumentation in Critical Discourse Analysis: \\ Deliberation and the Dialectic of Enlightenment
}

Isabela Fairclough et Norman Fairclough

Traduction : Sivan Cohen-Wiesenfeld

1 Cet article développe un travail récent sur le discours politique (Fairclough \& Fairclough 2011, 2012), qui articule l'analyse critique du discours (CDA) à la théorie de l'argumentation. Il part d'une conception de la politique au sein de laquelle la délibération, la décision et l'action constituent des concepts cruciaux. La politique consiste à décider de ce qu'il faut faire, et des lignes d'action à adopter dans des contextes de désaccord, de conflit d'intérêts et de valeurs, d'inégalité de pouvoir, d'incertitude et de risques. Ce processus est de nature essentiellement argumentative. Il implique la production d'arguments pratiques, de même qu'une démarche qui permet d'évaluer le poids des arguments mis dans la balance, à savoir une délibération. Nous avons développé notre approche à travers une étude et une analyse des réactions politiques, en Grande-Bretagne, à l'actuelle crise financière et économique.

2 La CDA a pour but d'étendre au discours les formes de critique connues dans le domaine de la science sociale critique. Comme nous le montrons dans Fairclough \& Fairclough (2012), nous considérons la théorie et l'analyse de l'argumentation comme potentiellement aptes à accroître la capacité de la CDA à atteindre ce but tant au niveau de la critique normative, que de la critique explicative. Notre approche de l'argumentation est en effet normative autant que descriptive, et nous considérons 
l'évaluation de l'argumentation pratique comme un pont jeté entre la théorie et l'analyse argumentatives et la CDA dans la mesure où elle s'intéresse aux diverses formes de critique du discours.

3 Par rapport à nos précédentes publications, cet article développe le raisonnement élaboré en faveur de l'incorporation, dans notre propre approche de la CDA, de la théorie et de l'analyse de l'argumentation et, plus spécifiquement de notre conception innovante de la délibération et de l'argumentation pratiques. Nous soutenons en particulier qu'une approche normative de l'argumentation est essentielle pour intégrer l'analyse du discours argumentatif à la critique, en présentant notre propre approche de la normativité. Nous avançons aussi que notre version de la CDA, enrichie par la théorie et l'analyse de l'argumentation, est en parfaite cohérence avec la théorie de l'agir communicationnel de Habermas et en particulier avec sa conception de la "constitution d'un horizon de sens", du potentiel d'« ouverture au monde " du langage et de sa « dialectique des Lumières ».

\section{Analyse critique du discours et science sociale critique}

4 La science sociale critique a pour but non seulement de décrire des sociétés, mais également de les évaluer par rapport à l'idéal de ce qu'elles devraient être afin de cultiver le bien-être de leurs membres. Cette évaluation est liée au souci de comprendre quelles sont les possibilités d'effectuer des changements susceptibles d'améliorer les sociétés en la matière, et quels sont les obstacles qui s'y opposent.

5 La science sociale critique a depuis longtemps reconnu l'importance des idées et des concepts dans la vie sociale. La réalité sociale est "conceptuellement médiatisée » (Marsden 1999) : en plus des évènements sociaux (comportement, pratiques), il existe toujours des idées de ces évènements (concepts, représentations, théories) qui sont produites par la vie sociale et ses effets, et qui ont en retour des conséquences sur la vie sociale ; elles contribuent aussi bien à maintenir les formes existantes qu'à les modifier. Et puisque les idées (concepts, représentations, théories) se manifestent à travers des types et des formes de discours particuliers (et que différentes idées, par exemple, de la justice, se manifestent dans ces différents discours), cette assertion peut être étendue au discours: les types et formes de discours existant doivent être expliqués socialement, et la vie sociale doit de son côté être en partie expliquée en termes d'effets de discours (Fairclough 1992, 2010, Fairclough \& Graham 2002).

6 L'analyse sociale critique comprend une critique de certains aspects de la vie sociale. Nous distinguons entre deux formes de critique : normative et explicative. La critique normative évalue les réalités sociales en les mesurant aux valeurs nécessaires à l'existence d'une "bonne société », globalement centrée sur sa contribution au «bienêtre " humain, considéré par exemple en termes de "respect du droit moral au développement des aptitudes humaines" (Nussbaum 2000 : 83). La critique explicative cherche à expliquer pourquoi les réalités sociales sont ce qu'elles sont, et comment elles se maintiennent ou se modifient. La critique normative traite de l'évaluation du comportement, des actions et des pratiques sociales comme, par exemple, de ce qui est juste ou injuste, de ce qui est équitable ou de ce qui est de l'ordre de l'exploitation, de ce qui est ou non raciste, et des croyances comme le vrai ou le faux. La critique explicative cherche à expliquer, par exemple, pourquoi et comment certaines réalités 
sociales perdurent malgré leurs effets dommageables. Elle tente de comprendre ce qui fait qu'un ordre social donné fonctionne, démarche évidemment nécessaire pour le changer dans le but d'améliorer le bien-être de l'homme (Sayer 2011). Ces deux formes de critique peuvent être étendues au discours, ce qui est l'objectif de la CDA. La critique normative inclut par exemple la critique du discours manipulatoire lorsque celui-ci fait partie intégrante d'une forme de domination. La critique explicative comprend à la fois des explications des types et formes particuliers de discours en tant qu'effets de causes sociales, et celles de l'établissement, de la continuation ou du changement d'un ordre social considéré comme étant partiellement un effet du discours. Une partie des sujets traités concernent les idéologies, c'est-à-dire les idées, les croyances et les préoccupations manifestées dans les discours qui contribuent à l'établissement, au maintien et à la reproduction des ordres sociaux et des relations de pouvoir.

7 La CDA ne peut mettre en pratique par elle-même une critique normative ou explicative, mais elle peut contribuer à se focaliser sur le discours et sur les relations qu'il entretient, de même que d'autres éléments sociaux - avec une critique interdisciplinaire. La CDA est un ensemble d'approches qui travaillent à étendre l'analyse sociale critique au discours; elles sont parfois très différentes et, à certains égards, incompatibles. Notre démarche dans ce domaine a été élaborée dans une série de publications qui incluent Fairclough 1989, 1992, 2000a, 2003, 2006, 2010.

Le discours est un élément social, une partie ou un aspect de la vie sociale dialectiquement relié aux autres (Fairclough 2001, Fairclough 2010): même si les analystes sociaux considèrent qu'il est nécessaire d'établir une distinction entre certains d'entre eux, ils ne sont pas complètement séparés les uns des autres. Par exemple, les économies néo-libérales sont apparues tout d'abord comme un ensemble d'idées et de discours néo-libéraux, qui se sont transformés avec succès (dans des circonstances et des conditions favorables) en de nouvelles réalités économiques, les économies néo-libérales. Il serait tout à fait erroné de dire que les économies néolibérales ne sont que des idées ou des discours dans la mesure où elles possèdent un caractère en partie matériel. Mais elles n'en sont pas moins en partie idées et discours : leurs caractéristiques matérielles sont un ensemble d'idées et de discours "rendus réels » et "opérationnels» (voir plus loin). La CDA ne se soucie pas uniquement de l'élément sémiotique, elle cherche également à travailler de manière interdisciplinaire (par exemple avec des spécialistes d'économie politique) afin d'identifier et de comprendre les relations entre éléments sémiotiques et matériels. La nature de telles relations varie selon les institutions et les organisations et suivant les différents lieux, et peut changer avec le temps; elle doit être établie par l'analyse.

9 La vie sociale peut être conceptualisée et analysée comme une interaction entre trois niveaux de réalité sociale: les "structures", les «pratiques» et les «évènements" sociaux (Chouliaraki \& Fairclough 1999). La relation entre les structures et les événements sociaux n'est pas considérée ici comme directe, mais comme médiatisée par des pratiques sociales qui sont des manières d'agir, de représenter et d'être, relativement stables et durables (bien que modifiables) - telles les pratiques de discussion et de débat politiques publics dans lesquelles on débat des réponses possibles à la crise. Les pratiques façonnent les évènements mais ne les déterminent pas, et les transformations du caractère des évènements peuvent, en s'accumulant, conduire à un changement des pratiques, susceptible à son tour d'amener des transformations structurelles. 
10 Toutes les structures, pratiques et évènements possèdent un caractère partiellement discursif ou sémiotique. Les évènements, dans leur aspect sémiotique, sont des «textes" (parlés, écrits, électroniques). Dans le cas des pratiques, les manières d'agir incluent les " genres "; les moyens de représentations comprennent les « discours », et les façons d'être incluent les "styles». Genre, discours et style sont des catégories sémiotiques. Ils présentent une certaine dose de stabilité au fil du temps. Les genres sont des manières d'agir et d'interagir, comme les interviews d'actualités ou les entretiens d'embauche, les reportages journalistiques ou les publicités. Les discours sont des moyens de représenter des aspects du monde qui peuvent généralement être identifiés avec les positions ou les perspectives de divers groupes d'acteurs sociaux (les partis politiques, par exemple). Les styles sont des manières d'être, des identités sociales, dans leur aspect sémiotique; par exemple, être un bon directeur signifie en partie savoir développer le bon style. Les champs sociaux, les institutions et les organisations sont constitués de pratiques sociales multiples reliées entre elles en forme de réseaux, dont la dimension sémiotique est un "ordre du discours", une configuration de genres, de discours et de styles différents (Fairclough 2000a). La politique, par exemple, est un champ social constitué par un réseau de pratiques sociales (associées avec des partis politiques, le fonctionnement du parlement, des élections, de la sphère publique etc.) incluant divers genres (le débat parlementaire, les interviews, les discours politiques, etc.), divers discours (politiques) et divers styles (de dirigeants, par exemple).

11 Les discours qui trouvent leur origine dans un champ social ou une institution particuliers (par exemple le discours économique néo-libéral, qui provient de la théorie économique universitaire) peuvent être "recontextualisés» dans d'autres (par exemple dans le domaine commercial, politique ou éducatif). Ils peuvent aussi naître dans un lieu ou un pays spécifiques, et être recontextualisés dans un autre. De plus, les discours peuvent, sous certaines conditions, être "rendus opérationnels » ou mis en opération, en pratique : ils peuvent être «représentés » comme de nouveaux moyens d'action et d'interaction, "inculqués" comme de nouvelles manières d'être (de nouvelles identités), et être matérialisés physiquement, par exemple comme de nouvelles façons d'organiser l'espace, notamment dans l'architecture. La représentation et l'inculcation peuvent eux-mêmes revêtir des formes sémiotiques : un nouveau discours sur la gestion (par exemple le discours de la "nouvelle gestion publique ») peut être représenté comme un ensemble de procédures de gestion incluant de nouveaux types d'interaction entre les dirigeants et les travailleurs, ou être inculqué comme un système d'identités qui incluent sémiotiquement les styles d'un nouveau type de dirigeants publics. Il faut souligner que ces procédures de mise en pratique ne sont pas inévitables : ce sont des possibilités contingentes, qui dépendent d'une combinaison de facteurs et de conditions à la fois matériels et sémiotiques (Fairclough, Jessop \& Sayer 2004).

12 Notre version de la CDA a été utilisée dans le cadre d'une collaboration interdisciplinaire entre divers domaines et théories (voir la gamme de celles-ci dans Fairclough 2010) y compris, depuis peu, dans «l'économie politique culturelle » (CEP, Jessop 2004, 2008, Jessop \& Sum 2001). Pour la CEP, les systèmes, institutions, relations et pratiques économiques et politiques sont des constructions sociales dont le discours constitue la dimension culturelle. Elle distingue entre les structures et les stratégies, celles-ci étant considérées comme passant au premier rang en temps de crise, lorsque 
les structures existantes ne fonctionnent plus de manière adéquate, et que prolifèrent les différentes stratégies des agents sociaux pour les transformer suivant des orientations particulières. Ces stratégies ont une dimension sémiotique : elles incluent des « imaginaires» de futurs états de choses que les agents sociaux cherchent à faire naître, par exemple des imaginaires économiques portant sur des manières d'opérer économiquement différentes de celles qui existent, et qui constituent des discours d'un type particulier. Certains de ces imaginaires seront, en termes de CDA, rendus opérationnels, matériels et réels, alors que la plupart ne le seront pas. L'une des principales orientations de recherche consiste à vérifier pourquoi et comment certains imaginaires et stratégies l'emportent sur d'autres, pour répondre, par exemple à la crise actuelle. La CDA fournit au CPE les moyens de traiter des questions sémiotiques, tandis que le CPE offre à la CDA une manière de conceptualiser l'analyse du discours dans une version d'économie politique qui manipule des dimensions matérielles et institutionnelles en même temps que la dimension sémiotique. Nous pensons que l'analyse de l'argumentation peut apporter une contribution significative au CPE en lui fournissant une approche systématique et cohérente permettant de rendre les catégories de stratégie et d'imaginaire du CPE opérationnelles pour l'analyse des textes (voir Fairclough \& Fairclough 2012, et plus loin dans le texte).

\section{La structure des arguments pratiques}

Les arguments pratiques portent sur ce qui doit être fait, par opposition aux arguments sur la nature du cas (arguments " théoriques»). La conclusion d'un argument pratique est une assertion normative ou prescriptive, affirmant qu'un agent doit réaliser une action, ou que l'action en question est recommandée. La structure du raisonnement pratique que nous suggérons est la suivante (Figure 1). L'hypothèse selon laquelle une action A pourrait permettre à l'agent d'atteindre ses objectifs (O), à partir des circonstances $(\mathrm{C})$ et en accord avec certaines valeurs $(\mathrm{V})$, conduit à la présomption qu'il doit accomplir A. Souvent, le contexte de l'action est considéré comme un problème (évalué négativement au vu des valeurs ou des préoccupations existantes de l'agent) et l'action comme la solution à ce problème.

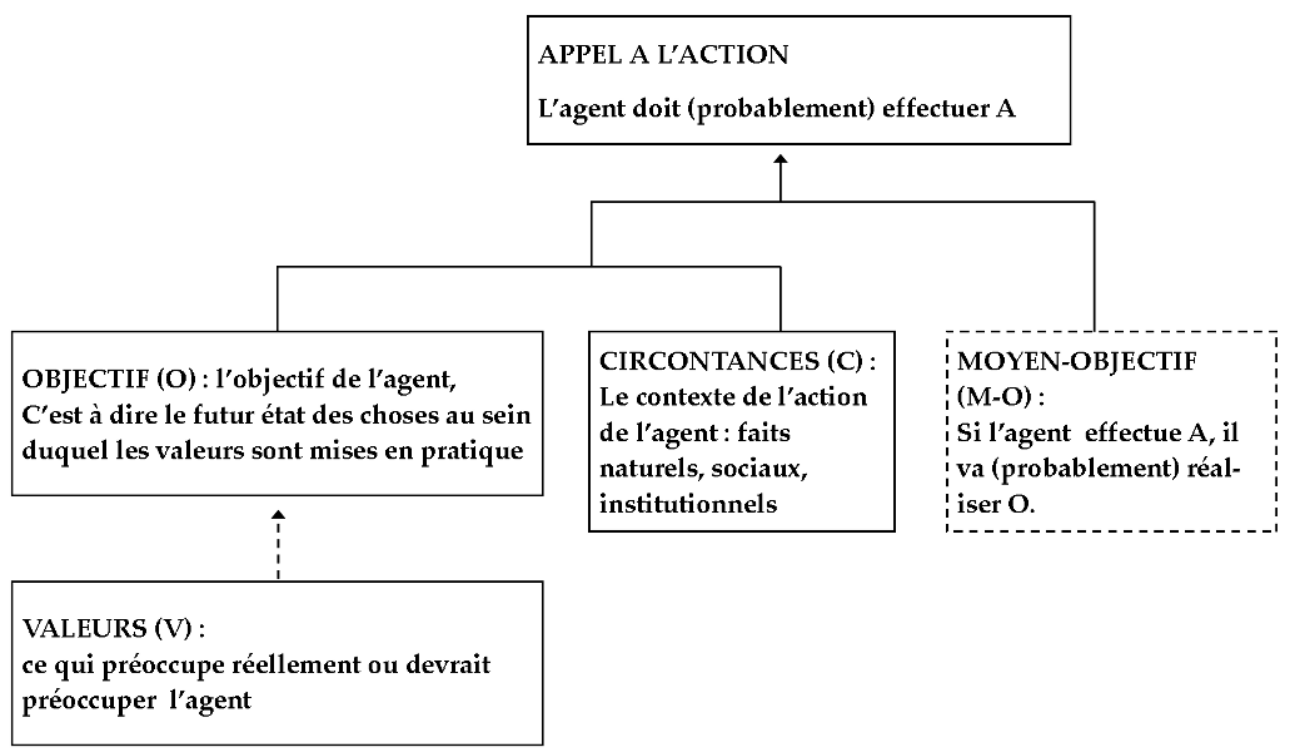

Figure 1 : La structure des arguments pratiques 

objectifs. Les agents combinent une connaissance des circonstances environnantes et de leurs objectifs avec une relation probable moyen-objectif susceptible de les conduire des circonstances dans lesquelles ils se trouvent, au futur état de choses qu'ils visent à atteindre. Les agents choisissent certaines actions plutôt que d'autres non seulement en fonction de leurs buts, mais également parce qu'ils se trouvent dans des circonstances particulières et non dans d'autres. Le contexte restreint l'éventail des actions envisageables et des choix possibles. L'action qui émerge comme étant (probablement) la bonne est supposée transformer l'actuel ensemble de circonstances de manière à l'adapter au but de l'agent, lui-même influencé par les valeurs qui sont les siennes (soit ses véritables valeurs, soit celles que lui-même - ou un autre argumentateur - pense qu'il devrait adopter). Ainsi, les circonstances vont être amenées à s'aligner, pour ainsi dire, sur la source de normativité sous-jacente à l'action.

Dans la perspective dialectique ${ }^{1}$ que nous adoptons, l'argumentation est un échange dialogique de mouvements entre deux interlocuteurs ou plus. L'argumentation implique un dialogue parce qu'elle survient toujours en réponse à quelque différence d'opinion, à un doute ou à une critique exprimés ou anticipés. Pour la pragmadialectique telle qu'elle a été développée par van Eemeren, l'objectif normatif du dialogue est la résolution d'une différence d'opinion d'une manière rationnelle, ou selon ses mérites, et l'argumentation est comprise comme une procédure destinée à tester l'acceptabilité d'un point de vue à la lumière d'un questionnement critique (van Eemeren \& Grootendorst 1992, 2004, van Eemeren 2010).

D'autres théories dialectiques sont également fondées sur l'idée que l'argumentation prend la forme d'un dialogue doté d'une fonction critique. Selon Walton, un dialogue est un type de conversation dirigée vers un objectif, à laquelle deux personnes (au moins) participent par des tours de parole; un dialogue argumentatif est une "séquence de mouvements reliés " entre eux (d'actes de langage) dans lesquels les participants posent des questions critiques et y répondent, pour tester la pertinence (à valeur expérimentale, provisoire) d'un argument et résoudre ainsi une différence d'opinion (Walton $2006: 2$ ).

relie à chaque schéma argumentatif un ensemble de questions critiques. Un schéma de raisonnement pratique peut être évalué en posant l'ensemble de questions critiques suivantes (2007 : 221-222) :

- Quels sont les autres objectifs de l'agent susceptibles d'entrer en conflit avec son objectif o ?

- Dans quelle mesure l'objectif s'appuie-t-il sur les valeurs de l'agent (ou du moins est compatible avec elles)?

- Quelles sont les lignes d'action alternatives susceptibles de conduire au même objectif endehors de celle qui est préconisée, qu'il faut prendre en considération?

- Parmi ces alternatives, quelle est la plus efficace pour atteindre l'objectif 0 ?

- Parmi ces alternatives, quelle est la meilleure compte tenu des valeurs de l'agent?

- Quelles sont les raisons permettant d'affirmer qu'il est possible de réaliser l'action en question sur le plan pratique dans une situation donnée?

- Quelles sont les conséquences de l'action susceptibles d'avoir une valeur négative plus importante encore que la valeur positive d'o qui doivent être prises en compte?

D'après Walton, le raisonnement pratique est révisable par nature. Dans des conditions d'information incomplète, d'incertitude et de risque, les agents sont forcés d'adopter 
une ligne d'action révisable qu'ils sont généralement prêts à modifier en fonction des conséquences susceptibles d'émerger, ou des changements qui interviennent dans le contexte de l'action. La conclusion d'un argument pratique est par nature sujette à révision en fonction d'un apport d'information nouveau, c'est-à-dire si l'action échoue d'une manière quelconque, si l'agent prend conscience d'un conflit de valeurs, ou d'un conflit avec d'autres objectifs qu'il poursuit ou de ceux d'autres agents. Les questions critiques révèlent divers problèmes possibles que pose l'argument. Selon Walton, il existe au moins deux manières principales de mettre un argument en doute : l'une est de "poser des questions qui soulèvent des doutes sur l'argument", l'autre de " proposer une réfutation ou un contre-argument ", ce qui est une forme d'attaque plus forte (2006:27). D'après nous, ce dernier type de mise en doute demande généralement d'évoquer les conséquences négativesde l'action proposée et de montrer qu'elles sapent ses objectifs et ses valeurs spécifiques, ou d'autres valeurs et objectifs qu'il importe de ne pas saper; par conséquent, ne pas effectuer l'action, ou s'abstenir d'agir, est plus raisonnable que d'exécuter l'action (Fairclough \& Fairclough $2012: 62-68$ ).

19 Si l'on admet que le raisonnement pratique peut par nature être mis en échec et qu'il est de l'ordre de la présomption, il en résulte que la revendication pratique en faveur d'une action ne peut être avancée que provisoirement. Le caractère raisonnable d'une proposition d'action peut toujours être mis en doute sur la base d'une information nouvelle: les circonstances changent, les agents réalisent qu'ils doivent prendre en compte d'autres objectifs et valeurs, ou bien l'action est susceptible d'avoir des conséquences imprévisibles et indésirables. Sans doute, les arguments pratiques sont souvent mis en avant avec un fort degré de certitude, mais ils peuvent conduire à une conclusion qui n'est certaine que dans la mesure où nous possédons toutes les données pertinentes, et seulement s'il n'existe pas de conflit d'objectifs ou de valeurs possible et que nous pouvons être sûrs que toutes les considérations possibles pointent vers une seule et même direction. De tels scénarios ne sont guère courants dans les circonstances quotidiennes. Cependant, dire que les arguments pratiques peuvent en principe être mis en échec ne signifie pas qu'ils sont par nature ou toujours faibles. Un argument pratique peut être rationnel et fort, et l'acceptation de ses prémisses peut constituer une bonne raison d'en adopter la conclusion, s'il est le fruit d'un processus de questionnement critique. A l'inverse, la raison pratique qui plaide en faveur de l'action proposée peut être affaiblie par l'incapacité de l'argumentateur à répondre d'une manière satisfaisante aux questions critiques, c'est-à-dire par l'échec de l'argumentation à tenir tête à la critique systématique des prémisses et de la thèse.

Le raisonnement pratique implique en général de " peser " les raisons pour et contre l'action proposée, et tout débat pratique qui suppose une telle évaluation des motifs est un exemple de « délibération ». Celle-ci peut être menée par une personne seule ou par un groupe ; elle peut s'effectuer dans un débat unique portant sur les raisons qui jouent pour ou contre une action spécifique, mais elle consiste souvent en un questionnement critique et une évaluation des arguments et des motifs pour et contre des lignes d'action alternatives. Une délibération peut être pratique ou non : on peut délibérer des moyens d'atteindre un but pris en lui-même comme une donnée, mais on peut également discuter des objectifs et même des valeurs en poursuivant un questionnement critique sur l'objectif et les valeurs-prémisses d'arguments particuliers, et en soupesant les objectifs et les valeurs alternatifs possibles. Le questionnement critique peut tester les prémisses d'un argument, ou l'induction des 
prémisses vers la conclusion, ou il peut remettre directement en question l'action proposée sur la base des conséquences susceptibles de saper l'objectif, ou d'autres objectifs vis-à-vis desquels l'agent s'est engagé.

\section{Un exemple : la délibération sur le discours budgétaire de Georges Osborne et la stratégie d'austérité du gouvernement de coalition}

21 L'actuel gouvernement de coalition britannique entre Libéraux-Démocrates et Conservateurs a, depuis mai 2010, entrepris de réduire le déficit budgétaire et la dette publique de manière draconienne. La nouvelle stratégie d'austérité fut d'abord annoncée dans le discours budgétaire du Chancelier Osborne en juin 2010 (HM Treasury ${ }^{2}$ 2010), dans lequel la nécessité de coupes budgétaires massives était justifiée par les effets catastrophiques que déclencherait tout échec à faire face à la dette, mais aussi par un souci d'équité et de prospérité future pour tous.

Reconstruisons les lignes principales de l'argumentaire d'Osborne dans ce discours ${ }^{3}$. L'affirmation principale est que le gouvernement doit immédiatement entreprendre de réduire rapidement le déficit budgétaire et la dette publique, principalement par une réduction des dépenses plutôt que par une augmentation des impôts. Dans les prémisses circonstancielles, on nous dit que nous sommes confrontés à une «situation d'urgence » que le gouvernement a " hérité de son prédécesseur ", aux « ruines d'une économie construite sur la dette publique ", et aux craintes suscitées par la persistance de la dette nationale (qui pourrait conduire à des taux d'intérêt paralysants). Dans les prémisses concernant les objectifs, le but à long terme est de «se relever des ruines d'une économie construite sur la dette publique et de construire une nouvelle économie équilibrée » qui ne dépende pas de façon excessive des services de la finance, au sein duquel "toutes les industries se développeront» et «la prospérité sera partagée par toutes les sections de la population et toutes les parties du pays", d'amener « un redressement durable du secteur privé ». A court terme, l'objectif est de « faire chuter la dette publique et arriver à une enveloppe qui équilibre le budget actuel d'ici la fin de la période parlementaire ». Osborne met en œuvre une stratégie : il ne s'agit pas d'une simple visée, mais d'un objectif à long terme et de divers sous-objectifs plus immédiats et à plus court terme, dont dépend la réalisation des objectifs à long terme. Dans les prémisses moyens-objectif, le procédé préconisé pour obtenir une réduction rapide de la dette publique et du déficit budgétaire est présenté comme nécessaire et suffisant à la fois pour atteindre l'objectif à long terme d'une économie durable et équilibrée. Dans les prémisses de valeurs, la "prudence financière » et la "responsabilité » sont évoqués en même temps que "l'équité", combinaison de valeurs évidente dans l'affirmation d'Osborne selon laquelle le budget « est dur, mais il est aussi équitable». Il est «inévitable» qu'il soit «dur pour le peuple», mais la «priorité » d'Osborne a été de «faire en sorte que les mesures soient équitables ».

Son argumentation présente un caractère délibératif. D'un côté, il rejette le contreargument selon lequel le gouvernement doit, non pas commencer par s'occuper d'abord de la dette et du déficit, mais «miser sur la croissance », en présentant les conséquences négatives qu'aurait le fait de ne pas réduire rapidement la dette et le déficit. Le gouvernement perdrait la «crédibilité » qu'il a commencé à gagner, avec 
comme résultat "une hausse des taux d'intérêt, une augmentation des faillites d'entreprises, un accroissement important du chômage, et même le risque potentiel d'une perte de confiance catastrophique et la fin de la relance ». Il donne également plusieurs arguments d'autorité : «l'opinion de la communauté internationale [...] exprimée lors de la dernière réunion du G20 [est que] les pays ayant de gros déficits fiscaux ont besoin d'accélérer le rythme de leur consolidation fiscale »; des preuves «rassemblées par l'Organisation pour la Coopération et de Développement Economique, le Fonds Monétaire International entre autres [...] ont montré que les consolidations obtenues par une baisse des dépenses sont plus efficaces pour corriger les déficits et relancer la croissance que celles obtenues par une augmentation des impôts »; « les prévisions [de l'office pour la Responsabilité budgétaire] prouvent [qu'il est possible d'atteindre nos objectifs]». Par ailleurs, il laisse entendre que les propositions et les arguments présentés par le gouvernement ont déjà fait l'objet de délibérations internes au sein de celui-ci. Par exemple, Osborne reconnaît que les coûts de la stratégie gouvernementale sont « lourds pour le peuple», mais il soutient que ceci est « inévitable » étant donné les circonstances, qu'une « catastrophe [...] s'ensuivrait » si on ne se préoccupait pas des dettes, et que sa priorité a été de "s'assurer que les mesures soient équitables ", que les plus riches paient plus que les plus pauvres.

Voici quelques fragments illustratifs tirés de la dernière partie de ce discours. Un extrait plus important et une analyse plus en profondeur se trouvent au chapitre 4 de Fairclough \& Fairclough (2012).

Monsieur le Président, je ne cache pas à cette Chambre que l'impact combiné des changements concernant les impôts et les avantages sociaux que nous introduisons aujourd'hui sont lourds pour le peuple. Cela est inévitable étant donné l'ampleur des dettes auxquelles notre économie est confrontée, et la catastrophe qui s'ensuivrait si nous échouions à y faire face. Ma priorité en élaborant ce budget a été d'assurer que les mesures soient équitables. Que toutes les parties de la population soient mises à contribution, mais que les plus riches paient plus que les plus pauvres. Pas seulement en valeur absolue, mais aussi en proportion du revenu. [...]

Au total, chacun paiera quelque chose, mais ceux qui sont situés au bas de l'échelle des revenus paieront proportionnellement moins que ceux qui se trouvent au sommet. Il s'agit d'un budget progressif.

M. le Président [...], aujourd'hui, nous entreprenons une action décisive pour gérer les dettes dont nous avons héritées et pour faire face au risque économique extrême auquel notre pays est exposé. Nous avons été durs, mais nous avons aussi été équitables. Nous avons posé les jalons d'un budget équilibré et d'une baisse de la dette nationale à la fin de la période parlementaire. Nous avons insisté pour que les quatre cinquième des montants nécessaires à la réduction de notre déficit soient pris sur les dépenses du gouvernement. Nous avons protégé l'investissement du capital de coupes supplémentaires et nous nous sommes attaqués à l'augmentation en flèche des coûts du système social. Nous avons posé les bases de la relance économique dans tous les secteurs de notre pays et l'avons doté d'impôts commerciaux qui comptent parmi les plus compétitifs au monde [...].

Malheureusement, en même temps que l'adoption inévitable de ce budget, nous avons dû augmenter les impôts. Nous avons dû payer la note de l'irresponsabilité du passé. Nous avons dû réapprendre la vertu de la prudence financière. Mais ce faisant, nous avons veillé à répartir équitablement la charge. Nous avons payé aujourd'hui les dettes des échecs passés et posé les fondements d'un avenir plus prospère - les plus riches payant le plus, et les plus vulnérables étant protégés. Voilà notre approche. La prospérité pour tous. Voilà notre objectif [...] 
upart des questions critiques portant sur l'argumentation pratique suggérées par Walton (voir plus haut), de même que d'autres suggérées par nous (Fairclough \& Fairclough 2012: 67), ont été posées dans le cadre du débat public sur la stratégie d'austérité gouvernementale et les arguments en sa faveur, reconstitués ci-dessus. Ainsi un questionnement critique en profondeur de la relation entre l'action proposée et les objectifs a été mené. Jean-Claude Trichet, président de la Banque Centrale Européenne, a affirmé que «le réajustement des dépenses publiques» (les mesures d'austérité) devait être « accompagné de réformes structurelles afin de promouvoir une croissance à long terme ", suggérant que les propositions du gouvernement étaient peut-être nécessaires, mais pas suffisantes (Trichet 2010). Le rédacteur économique du Financial Times, Martin Wolf, a par ailleurs soutenu que l'action proposée n'était pas (comme l'affirmait Osborne) nécessaire pour atteindre les objectifs à long terme, en partie parce que son affirmation selon laquelle la Grande-Bretagne devrait, sans cette action, faire face à un destin similaire à celui de la Grèce ou de l'Irlande, n'était pas fondée (Wolf 2010). La proposition a également fait l'objet d'un questionnement très critique quant aux conséquences : elles seraient susceptibles de saper les objectifs visés plutôt que de contribuer à les atteindre. Ainsi Martin Wolf a affirmé qu'« un rapide retrait du soutien fiscal réduirait non seulement l'actuel PIB, mais aussi la croissance éventuelle, par son impact négatif sur les investissements ", et pourrait donc nuire aux objectifs du gouvernement (Wolf 2010).

Le questionnement critique n'a pas été uniquement instrumental, considérant les objectifs comme des données pour n'examiner que les moyens de les atteindre; les objectifs eux-mêmes ont été remis en question. Une des orientations de ce questionnement a été de se demander si les objectifs déclarés étaient bien les véritables buts du gouvernement, ou la façade en trompe-l'œil d'un ordre du jour idéologique utilisant le déficit comme prétexte pour ramener le pays en arrière. Le rédacteur économique du Guardian, Larry Elliott, a affirmé que le budget d'Osborne montrait que son « programme véritable est d'achever le travail de démolition de l'Etat-Providence commencé dans les années 1980 » (Elliot 2010). Ceci équivaut à la thèse selon laquelle l'argumentation du gouvernement est une rationalisation: la raison qu'il donne en faveur de la politique qu'il préconise n'est pas la véritable raison pour laquelle il préconise cette politique même (Fairclough \& Fairclough 2012 : 95-99, Audi 2006).

Un autre axe de ce questionnement était centré sur l'idée que derrière les objectifs déclarés se cache un désir de "retour à la normale » (business as usual) plutôt que de se lancer dans la restructuration de l'économie nécessaire à une relance durable. Caroline Lucas, la députée du parti des Verts, a déclaré qu'au lieu « de tailler dans les dépenses publiques dans l'espoir du retour à la normale ", le gouvernement "doit saisir cette occasion pour reconfigurer le système économique totalement insoutenable qui a contribué à nous pousser vers l'effondrement financier, la crise de l'environnement et une insécurité croissante en matière d'énergie » (Elliott \& al. 2011). Les partisans de la restructuration radicale de l'économie préconisent dans de nombreux cas l'(objectif) imaginaire d'un "nouveau pacte vert». Les définitions du contexte de l'action dans le cadre des prémisses circonstancielles font également l'objet d'un questionnement critique, y compris sur les explications avancées par le gouvernement des causes de la crise, et sur le fait de tenir pour responsable de la dette et du déficit le gouvernement travailliste précédent plutôt que les banquiers et l'effondrement du système financier.

Argumentation et Analyse du Discours, 9 | 2012 

la plausibilité, en termes de raison, des valeurs sur lesquelles le gouvernement affirme s'engager, et la question de savoir si les buts et l'action proposée servent véritablement les valeurs déclarées. Sur ce point, le journaliste du Guardian Seumas Milne a avancé que les revendications d'équité d'Osborne dans le cadre du budget de juin sont frauduleuses; il cite des statistiques de l'Institut des Etudes Fiscales montrant qu'en 2015, l'impact du budget sur le dixième le plus défavorisé de la population sera cinq fois plus important que son impact sur les plus riches. Loin d'être progressif, comme le gouvernement le prétend, le budget est régressif, affirme-t-il, et il va frapper durement les plus pauvres; ce sont eux et les handicapés qui vont «payer le prix de la récession des banquiers ». En augmentant la TVA et en réduisant une grande partie des avantages sociaux, tout en diminuant les impôts sur les entreprises et en ne pénalisant que légèrement les banques par une "taxation que minimisent des bonus excessifs", Osborne a tourné la «prétention à l'unité sociale » du gouvernement «en une amère plaisanterie » (Milne 2010). Les arguments selon lesquels la politique du gouvernement n'est pas équitable sont parfois associés avec ceux qui prétendent que les politiques alternatives (par exemple, un programme de création d'emplois ou une taxe sur les transactions financières commerciales) seraient plus justes. L'analyse critique s'est également concentrée sur les divers sens du mot " équité ", comprenant l'équité selon le juste mérite, comme la protection des plus défavorisés, et comme le souci de justice intergénérationnel. Osborne fait appel à "l'équité selon le juste mérite " lorsqu'il affirme qu'il ne devrait plus être possible pour ceux qui choisissent de ne pas travailler de s'en tirer mieux que ceux qui travaillent, comme c'est souvent encore le cas, prétend-il. Mais quand on en vient à la question de savoir qui doit souffrir des coupures budgétaires, il soutient qu'il est juste que la charge en soit partagée par la population. La question critique qui se pose alors est: est-il compatible avec le principe d'équité (selon le juste mérite) de forcer la population à payer pour une crise qu'elle n'a pas déclenchée, tout en demandant si peu aux banques et aux banquiers qui en sont l'origine et devraient, par conséquent, selon la même logique, payer pour les dommages?

L'équité est présentée dans le discours sur le budget et partout ailleurs comme à la fois une préoccupation active du gouvernement (qui souhaite être équitable) et un fait institutionnel (les gouvernements ont la tâche de l'être). Le gouvernement a clairement intérêt à montrer qu'il reconnaît son devoir d'équité, et à affirmer qu'il veut le remplir. En effet celle-ci, en tant que valeur publiquement partagée, bénéficie d'une légitimité indiscutable, et les appels à l'équité sont susceptibles d'augmenter l'acceptabilité rhétorique et dialectique de l'argument. Les gouvernements doivent essayer de convaincre les gens qu'ils reconnaissent et adoptent le principe d'équité, car une perception du contraire assez répandue parmi la population risquerait de porter préjudice à leur légitimité. Ceci explique pourquoi la remise en cause critique des prétentions d'équité du gouvernement joue un rôle primordial dans le débat public.

L'équité peut difficilement être contestée en tant que valeur rationnellement acceptable, mais la remise en question de son acceptabilité rationnelle peut être dirigée, comme nous l'avons indiqué, vers l'incohérence ou l'illogisme des divers sens dans lesquels la notion est employée, ainsi que la relation entre l'équité et d'autres valeurs. Meacher (2010b) observe que les Conservateurs comme les Nouveaux Travaillistes ont, avec un relatif succès, mis en avant la valeur d'équité dans leur ordre 
du jour. Pourtant, il est difficile de voir comment celle-ci peut être compatible avec les énormes inégalités de richesse et de revenu qui ont inéluctablement augmenté sur une période de trente ans et continuent à croître pendant la crise. L'acceptabilité rationnelle des prémisses de valeur dépend de la manière dont les différentes valeurs sont évaluées et reliées entre elles, et les relations entre ces valeurs constituent un sujet clé tout à fait pertinent du questionnement critique.

31 Comme nous l'avons brièvement indiqué (voir chapitre 4 de Fairclough \& Fairclough 2012 pour un compte rendu détaillé), le débat public sur la stratégie d'austérité du gouvernement en réponse au budget d'Osborne présente un caractère non instrumental. Il n'examine pas seulement les moyens proposés pour atteindre les buts visés, mais également ces objectifs eux-mêmes, les valeurs qui les sous-tendent, et la définition du contexte de l'action et ses explications. Par ailleurs, il ne se contente pas de remettre en question l'acceptabilité rationnelle des prémisses et les liens inférentiels entre celles-ci et la proposition, ni de produire des contre-arguments qui récusent la proposition elle-même : il forge également des prémisses alternatives, des liens inférentiels et des propositions. On pourrait dire que le questionnement est critique dans un sens négatif et positif à la fois. Le débat peut être positif et créatif en particulier par l'apport, sur la scène publique et dans le champ du questionnement et de l'évaluation critique, de nouveaux objectifs, de nouveaux imaginaires, de nouvelles conceptions des valeurs et de leur interrelation, de nouvelles analyses et explications du contexte de l'action. Nous voudrions arguer que la force de notre conception particulière de l'argumentation et de la délibération pratiques est qu'elle peut s'adapter à cet aspect positif et créatif du débat. Dans une dernière partie, nous suggérerons que cette caractéristique contribue à sa capacité à concourir à la promotion des objectifs de la science sociale critique et de la CDA.

\section{Les théories de l'argumentation et les objectifs de la CDA et de la science sociale critique}

Dans cette section finale, nous développerons l'argument présenté dans Fairclough \& Fairclough (2012), qui vise à intégrer dans la CDA la théorie et l'analyse de l'argumentation, et plus spécifiquement l'approche innovante de l'analyse et de l'évaluation de l'argumentation et de la délibération pratiques que nous proposons.

Notre premier argument en faveur de cette intégration provient de considérations sur la nature du politique et du discours politique. Faire de la politique, nous l'avons dit, consiste essentiellement à opérer des choix sur la manière d'agir en réponse à des circonstances et des évènements, à la lumière de certains objectifs et valeurs; choix fondés, ainsi que les actions qui en découlent, sur un raisonnement pratique portant sur ce qui doit être fait. C'est de cette manière qu'Aristote caractérise la politique dans son Ethique à Nicomaque: comme une action, fondée sur des décisions qui résultent d'une délibération. Nous considérons donc le discours politique essentiellement, quoique que bien évidemment pas exclusivement, comme de l'argumentation, et plus spécifiquement comme de l'argumentation et de la délibération pratiques. Par conséquent, l'analyse du discours politique requiert des modèles d'analyse (critique) du discours qui inclue des méthodes claires d'analyse du discours argumentatif. 
34 Néanmoins, nous développons également un autre argument, à partir de ce que nous considérons comme une faiblesse de la CDA dans son ensemble, y compris la version avec laquelle nous travaillons, et qui porte sur le discours politique aussi bien que sur d'autres types de discours. Il remet en cause la tendance de la CDA à se concentrer essentiellement sur des représentations, et sur les discours comme des manières de représenter le monde. Nous considérons que l'analyse de l'action et des genres l'emporte sur l'analyse de la représentation et des discours, parce que les représentations reçoivent une signification critique en tant qu'éléments d'un type particulier d'action, qu'il s'agisse d'une narration, d'une explication, d'une argumentation théorique ou pratique. Or, les représentations ont souvent été isolées de l'action dans les analyses de la CDA. Dans le cas de l'argumentation pratique, nous considérons les représentations comme éléments constitutifs des arguments, comme prémisses des arguments qu'avancent les agents sur ce qu'il convient de faire.

L'une des plus importantes conclusions de notre travail sur le discours politique est que les discours (comme moyens de représentation) fournissent aux agents des mobiles d'action. En raisonnant pratiquement, les agents font appel à des discours qui sont associés à certains ordres structurels, institutionnels et moraux, qui leurs fournissent ainsi des raisons d'agir. Celles-ci sont des mobiles extérieurs, qui contraignent l'action indépendamment du désir des agents, bien qu'ils puissent être, et c'est souvent le cas, intériorisés par eux sous forme de désirs et de préoccupations. Les agents peuvent être conscients de la nature externe de ces motifs d'action, ils peuvent les accepter sans les adopter, ou encore ne pas en tenir compte ou leur résister. Ils peuvent au contraire ne pas être conscients de la nature externe de ces mobiles, les interpréter ou les présenter faussement comme des raisons purement internes, émanant de leurs propres préoccupations et désirs - un scénario typique de l'idéologie. Notre proposition de traiter les représentations et les discours auxquels ils sont associés comme des éléments constitutifs (prémisses) des arguments est par conséquent, à nos yeux, cruciale pour arriver à un traitement satisfaisant de la dialectique structure-agent : le raisonnement pratique est une interface entre les structures et l'agentivité, qui rassemble les contraintes des unes avec les motivations de l'autre.

Un autre trait important de notre approche est qu'elle fournit une explication cohérente de l'opérationnalisation (représentation, inculcation, matérialisation) du discours, et plus spécifiquement de la manière dont des représentations particulières du monde peuvent, dans certaines conditions, avoir des effets constructifs sur le monde, et contribuer à sa transformation selon des orientations particulières. Si de telles représentations sont associées, comme nous l'avons suggéré, avec des discours qui fournissent aux agents des mobiles externes d'action, on comprend alors comment elles peuvent modeler les conclusions (propositions) de l'argumentation pratique et de la délibération, susceptibles à leur tour d'influencer les décisions et l'action. Les représentations du monde peuvent avoir sur les agents des effets constructifs en réactualisant les actions qui le transforment. De telles représentations peuvent consister en des imaginaires qui servent de prémisses de but, qui portent en eux des états de choses futurs (possibles et souhaitables) comme autant d'alternatives aux situations existantes, et que l'action stratégique tend à réaliser.

Prenons un exemple dans le discours sur le budget d'Osborne. Nous avons noté que l'une des valeurs à laquelle font appel les prémisses de son argumentation est « l'équité », mais que la « prudence financière » et la « responsabilité » sont invoquées à 
ses côtés. Osborne prétend avoir préservé un équilibre entre ces valeurs potentiellement conflictuelles : le budget est "dur », mais il est aussi «équitable ». L'équité est interprétée comme le fait pour les personnes de recevoir des récompenses ou de supporter des coûts en fonction de leur "juste mérite ", mais également comme le fait que les coûts (par exemple ceux de la crise) soient répartis parmi l'ensemble de la population, toutefois selon les capacités de chacun à y faire face. Cependant, «l'équité » n'est pas interprétée comme le fait d'atteindre ou d'augmenter l'égalité (de richesse, de revenu etc.) entre les gens, bien que pour certains participants du débat public, les inégalités énormes et croissantes existant en Grande-Bretagne, et qui ont continué de croître pendant la crise, soient incompatibles avec « l'équité ». On peut dire qu'Osborne présente « l'équité » d'une manière particulière, et que d'autres participants au débat la représentent différemment, qu'il existe différents discours sur «l'équité ». Celui d'Osborne fournit des raisons d'agir d'une certaine manière, d'autres donneraient des raisons d'agir différemment (par exemple pour réduire les inégalités de richesse et de fortune). Il s'agit de discours externes, bien qu'Osborne prétende implicitement avoir intériorisé le sien, c'est-à-dire qu'agir pour atteindre « l'équité » dans ce sens est à la fois ce qu'il désire, et ce qui est juste. "L'équité » comme valeur en faveur de laquelle les gouvernements doivent agir est ancrée dans les institutions politiques des sociétés démocratiques et est reconnue comme un test de légitimité. Le discours spécifique d'Osborne sur l'équité est externe d'une manière différente, il appartient à une tradition politique particulière. Dans la mesure où ces discours fournissent des raisons externes d'agir qui sont acceptées comme raisonnables, ils peuvent avoir des effets constructifs sur le monde en réactualisant les actions qui le transforment. L'équité en elle-même n'est pas controversée, mais le discours particulier d'Osborne sur l'équité l'est, et est susceptible d'être moins bien reçu, par exemple, dans la mesure où l'on remarque la contradiction que nous avons notée ci-dessus dans l'application de l'interprétation de "l'équité » comme obtention de récompense par certains ou prise en charge des coûts en fonction de leurs « justes mérites».

Notre approche de l'argumentation, nous l'avons dit, est normative. Tant la pratique de l'argumentation que celle de la politique (nous soutenons, bien sur, que cette dernière est essentiellement argumentative) présentent un caractère normatif, dans le sens où l'évaluation par les participants des arguments et des actions politiques d'autrui font partie intégrante de ces pratiques. Dans le discours politique, les participants sont constamment en train d'évaluer et de remettre en question les arguments d'autrui, et nous avons en effet défini la délibération précisément en ces termes. Notre position devient plus controversée lorsque nous affirmons qu'il ne suffit pas pour les analystes de décrire comment les agents évaluent les arguments et d'identifier les normes qu'ils appliquent pour ce faire : ils doivent également se demander si leurs évaluations sont raisonnables, c'est-à-dire qu'ils doivent évaluer les évaluations des participants. Par conséquent, notre approche englobe deux sortes de normativité: la normativité " profane » des participants, et la normativité analytique des analystes. Si les analystes ne pratiquaient pas ce deuxième type d'évaluation, ils ne décriraient pas correctement les pratiques argumentatives, car c'est un fait que les agents évaluent les arguments de manière non justifiée, sans voir leurs insuffisances, ou en voyant des lacunes qui n'existent pas, et se laissent persuader par des arguments qui ne sont pas pertinents. Par exemple, dans nos commentaires sur le discours d'Osborne, nous avons effectivement évalué comme justifiées les critiques des commentateurs qui l'ont perçu comme incohérent lorsqu'il a interprété la norme « d'équité » comme « juste mérite ». 
39 Nous avons distingué des motifs d'action internes et externes, mais les normes des modèles analytiques normatifs pour l'évaluation des arguments (et leur évaluation par les participants) ne sont pas " externes » dans le sens où elles seraient extérieures aux pratiques d'argumentation réelles. Au contraire, on peut toujours trouver des participants qui évaluent les arguments sur la base des mêmes normes que les analystes, et il n'y a pas de standard d'évaluation qui soit exclusif aux analystes. Les modèles normatifs sont dérivés des normes que les participants appliquent en évaluant les arguments, mais ils généralisent à partir d'exemples et de contextes d'évaluation particuliers afin d'identifier des principes d'évaluation, et ils systématisent ces principes en les reliant avec une théorie de la rationalité. Les agents n'appliquent pas toujours ces principes d'évaluation de manière logique et complète, bien qu'il soit prouvé qu'ils les reconnaissent comme raisonnables (van Eemeren \& al. 2009).

40 A nos yeux, le caractère normatif de l'approche est essentiel pour sa capacité à promouvoir les objectifs de l'analyse sociale critique et de la CDA. L'argument général est que, dans la mesure où la critique possède un caractère normatif inhérent, l'analyse de l'argumentation (et l'analyse du discours en général) qui aspire à faire avancer les objectifs de la critique, doit elle-même être normative. Mais il existe également un argument plus spécifique, lié aux processus d'apprentissage qui se présentent comme les conditions préliminaires à tout développement de la rationalitésusceptible de rehausser sa capacité critique (nous reviendrons sur ce problème plus loin). Alors que les modèles normatifs puisent leurs standards dans la pratique argumentative réelle, la généralisation et la systématisation qu'ils effectuent peuvent elles-mêmes contribuer à l'apprentissage social et au développement de la pratique argumentative réelle. En rassemblant l'ensemble des possibilités de délibération de manière systématique (sous la forme d'une liste exhaustive de questions critiques possibles, par exemple), nous pouvons montrer plus clairement en quoi la pratique réelle ne réalise pas ce qu'elle pourrait et devrait être, et ce faisant, indiquer plus clairement des manières de l'améliorer, fournir des modèles pour le faire, et ainsi contribuer (dans des conditions favorables) au développement du potentiel critique de raisonnement. Concrètement, par exemple, nous suggérons que le fait de montrer de cette manière systématique comment la délibération et le débat publics sur les réponses politiques à la crise actuelle restent en-deçà de ce qui est possible et souhaitable (et parfois réalisé) pourrait, dans des circonstances favorables, aider à améliorer la pratique actuelle, à tirer des conclusions et à prendre des décisions plus raisonnables (et plus démocratiques) sur la stratégie et la politique à adopter.

41 On peut ainsi montrer qu'un ensemble systématique de questions critiques doit inclure la question de savoir dans quelle mesure les valeurs particulières inhérentes aux prémisses sont reliées à d'autres types de valeurs. Dans l'exemple tiré du discours d'Osborne discuté ci-dessus, la relation entre «équité » et « égalité » est ignorée ; ce que les débats sur la stratégie d'austérité tendent d'ailleurs généralement à faire. En éclairant la question cruciale mais souvent négligée de la manière dont les différentes valeurs s'équilibrent entre elles, il devient possible de contribuer à approfondir le débat public qui entend effectuer un examen critique des prémisses de valeurs fournissant des raisons d'agir, en dépassant les simples appels à «l'équité » qui peuvent mener à des concessions inconsidérées sur ce qui doit être considéré comme légitime.

Nous pensons également que la manière particulière dont nous proposons d'incorporer l'analyse et l'évaluation de l'argumentation dans la version de la CDA que nous utilisons 
met en valeur les caractéristiques dialectiques de celle-ci, qui contribuent de manière significative à sa capacité à promouvoir les objectifs de la théorie critique et de la science sociale critique ${ }^{4}$. Tout en arguant de façon polémique que la "philosophie de la praxis» (matérialisme historique) marxiste est réductrice lorsqu'elle établit une équation entre la praxis et le travail, et que la communication est également praxis, Habermas $(1984,1987)$ souhaite préserver le caractère dialectique de la philosophie de la praxis et du courant de la critique de la modernité qui relie Hegel, Marx et la théorie critique, qu'il oppose favorablement au caractère non dialectique du courant qui relie Nietzsche, Heidegger et le post-structuralisme (Derrida, Foucault). Dans une perspective dialectique, le potentiel communicatif et critique de la rationalité est donc considéré à la fois comme déformé (réduit à la raison instrumentalisée) et en même temps comme développé par la société moderne, alors que, dans la perspective non dialectique, la déformation de la raison est totale, et elle est donc soumise à une critique radicale, opposée à la critique dialectique. Dans la perspective dialectique, la critique de la raison (instrumentale) dans la société moderne vient de celle d'une rationalité plus large, qu'Habermas conçoit comme la rationalité de la communication qui se manifeste dans la recherche intersubjective de compréhension. La perspective non dialectique prend en considération ce qui constitue, dans une perspective dialectique, de fausses alternatives, qui sont plutôt des moments d'un processus dialectique unique : l'utilisation du langage d'une manière conventionnelle et normée, et l'usage créatif du langage dans une optique d'«ouverture au monde "; le monde vécu comme un arrière-plan opaque, et comme un subconscient dont nous pouvons prendre conscience ; la raison comme un pouvoir transcendant, et comme un masque du pouvoir. La perspective non dialectique refuse de reconnaître l'ambivalence de la vie moderne, et les possibilités de l'utilisation d'une dialectique éclairée et des développements de la philosophie des Lumières pour combattre ses distorsions, optant à la place pour une critique radicale dont les propres fondations normatives sont incertaines (Habermas $1987: 294-326)^{5}$.

La théorie de l'agir communicationnel s'expose aux accusations d'idéalisme, mais Habermas (2011 : 348-386) indique comment celles-ci peuvent être contrées.

De fait, c'est à travers les résultats et les conséquences qu'engendrent les actions finalisées au moyen desquelles interviennent dans le monde ceux qui appartiennent au monde vécu que celui-ci se reproduit matériellement. Mais ces actions instrumentales, en tant qu'elles représentent l'exécution de projets qui se rattachent - à travers les définitions communes de situations et à travers les processus d'intercompréhension - à ceux d'autres participants à l'interaction, sont donc intriquées à des actions communicationnelles [...], la reproduction symbolique du monde vécu est rétroactivement couplée à sa reproduction matérielle ${ }^{6}$.

La version de la CDA avec laquelle nous travaillons diffère des autres par les liens étroits qu'elle noue à la fois avec la perspective dialectique prônée par Habermas, et avec sa conception de l'interdépendance de la reproduction symbolique et matérielle du monde vécu. Cette version de la CDA conçoit et analyse la reproduction de la vie sociale (y compris sa transformation) comme un mécanisme au sein duquel les moments matériels et sémiotiques sont liés par un processus dialectique (Fairclough 2001, Fairclough, Jessop \& Sayer 2004). Elle considère en même temps le discours social comme ambivalent, c'est-à-dire caractérisé à la fois par une adhésion (souvent non réflexive) à des normes et des pratiques récusables, et aux moyens de les contester et de les transformer. Ce qu'elle peut apporter à la théorie critique, comme par exemple la théorie de l'agir communicationnel d'Habermas, ce sont des méthodes rigoureuses et 
systématiques d'analyse du discours social qui, en raison de leurs liens étroits avec les concepts et les préoccupations clés de la théorie critique, peuvent mener les perspectives critiques à avoir une portée sur les corpora discursifs réels, comme par exemple l'argumentation et le débat délibératif associés aux réponses à la crise actuelle. Elles peuvent contribuer ainsi à l'analyse critique de la dialectique du matériel et du symbolique (sémiotique) qui est appliquée à des cas particuliers de reproduction et de transformation sociale.

Mais nous arguons également que notre manière d'incorporer l'analyse de l'argumentation et son évaluation à la CDA augmente la capacité de celle-ci à le faire. Dans la perspective dialectique, la relation entre le langage comme "production constitutive d'horizon de sens » et comme pratique déterminée par des structures, est médiatisée par l'apprentissage. Habermas formule ceci de la manière suivante, au sujet de la philosophie de la praxis (matérialisme historique) :

C'est le monde des idées qui rend possible certaines interprétations d'une nature qui, sur cette base, devient l'objet de la coopération sociale ; or ce monde des idées est alors, à son tour, influencé par les processus déclenchés par le travail social. Au contraire de l'historicisme linguistique qui hypostasie la capacité du langage à ouvrir au monde, le matérialisme historique [...] table sur un rapport dialectique entre, d'un côté, les structures des conceptions du monde - qui autorisent la pratique intramondaine à travers une compréhension préalable du sens, et de l'autre, les processus d'apprentissage qui se traduisent dans le changement de structures des conceptions du monde $(2011: 378)$.

Le potentiel cognitif, et donc d'ouverture au monde, est néanmoins en développement : la rationalisation du monde vécu implique une actualisation du potentiel de raison propre à l'activité communicationnelle (2011: 386). Habermas fait référence à la "découverte hégélienne" selon laquelle il serait impossible de connaître, sans les dépasser, les limites historiques de la raison centrée sur le sujet (instrumentale) (2011: 360), ouverture au monde conditionnée par les processus d'apprentissage correspondant à une étape particulière de développement (ou «libération») du potentiel de rationalisation de l'action communicative.

Nous suggérons que notre conception de la délibération est fortement compatible avec l'approche dialectique, en ce qu'elle fournit des moyens de montrer comment l'apprentissage peut permettre la réalisation du potentiel de "constitution d'un horizon de sens » ou d'« ouverture au monde » du langage, comme condition préalable à la transformation sociale, dans le discours réel. Nous faisons ici référence à un débat concret que nous avons présenté dans Fairclough \& Fairclough (2012), en faveur d'une compréhension plus ample du processus de délibération qui permettrait de développer cette pratique dans le contexte de la crise actuelle: l'une des causes largement reconnue de la crise est la pauvreté des processus de délibération dans les sphères raréfiées de la haute finance et des autorités financières, au sein desquelles des décisions qui se sont avérées désastreuses ont été prises, en particulier, sans qu'aient été pesées de façon adéquate les conséquences négatives des actions fondées sur ces mêmes décisions. L'une des leçons claires de la crise est que les décisions ayant des conséquences importantes pour l'ensemble de la société exigent une profonde amélioration des processus de délibération: des processus au cours desquels le potentiel de questionnement critique rigoureux et créatif et de mise à l'épreuve des arguments, comme base de l'apprentissage et de la pensée créative ouvrant sur des 
horizon de sens et des imaginaires nouveaux, serait plus pleinement et plus largement développé.

Fairclough (2004/2011) tente de combler une lacune dans notre précédent travail sur notre version de la CDA, concernant l'analyse discursive des aspects sémiotiques du processus d'apprentissage comme un élément de transformation sociale. Alors que la CDA s'est focalisée sur l'interdiscursivité des textes et sur les changements sémiotiques comme transformations interdiscursives susceptibles d'être occasionnellement opérationnalisés dans le cadre de modifications sociales plus larges, elle n'a pas suffisamment tenu compte du fait que les possibilités de changements sémiotiques dépendent de l'ouverture au processus d'apprentissage, elle-même conditionnée par la dialogicité relative des textes et des interactions - la mesure dans laquelle ils sont ouverts à la différence et orientés vers elle (Fairclough 2000b). Notre explication fournit une base pour évaluer un débat comme rationnel ou non, en partie par rapport à son ouverture et son orientation vers la différence - son aptitude à soupeser les différents arguments dans leurs divers aspects et propriétés. La plus grande partie des débats concrets sont bien sûr plus ou moins irrationnels à cet égard. On peut considérer le débat selon notre approche comme un genre caractérisé par un potentiel cognitif pouvant être réalisé de manière plus ou moins satisfaisante. Un débat rationnel dans ce sens pourrait par exemple contribuer à faire connaitre les conséquences négatives ou positives possibles des diverses lignes d'action alternatives préconisées par les arguments. Il ne s'agirait pas seulement de donner la préférence à l'un des arguments proposés par rapport aux autres, mais éventuellement de considérer qu'aucun des arguments offerts ne passent de manière satisfaisante l'examen critique de leurs conséquences, et de développer de nouveaux arguments plus acceptables. Le débat délibératif pourrait par conséquent, de par sa capacité à développer la connaissance, conduire vers une pensée créative et une ouverture vers de nouveaux horizons. L'examen critique des arguments et la connaissance qui peut en résulter est donc le moyen permettant la réalisation du potentiel du langage pour la constitution d'un horizon de sens et le processus d'ouverture au monde.

Mais le modèle particulier de délibération, et le modèle d'argumentation pratique qu'il fonde, est décisif pour tout ce qui concerne notre capacité à expliquer ce potentiel d'apprentissage et de constitution d'un horizon de sens. Par exemple, notre modèle de délibération diffère des autres en ce qu'il inclut une prémisse de circonstance. De même, notre modèle de délibération comprend un questionnement critique de la manière dont est représenté l'état des choses existant, le contexte de l'action. L'un des éléments de la représentation des circonstances est constitué par les explications, celles de la manière dont la crise est survenue dans le cas du matériau que nous avons analysé. Les arguments associés à la stratégie d'austérité du gouvernement de coalition tendent par exemple à décrire la crise comme une explosion de la dette publique, et à l'expliquer comme le résultat de l'irresponsabilité fiscale du gouvernement travailliste précédent. D'autres arguments la présentent comme une crise bancaire causée par les spéculations erronées des banques. En délibérant sur un éventail d'arguments, on peut les passer au crible de la critique et les mettre en balance pour voir comment ils représentent et expliquent la crise. Au cours d'une telle délibération, les argumentateurs peuvent présenter une vision de ce qu'ils considèrent comme une explication rationnelle, qui fasse paraître comme irrationnelles celles proposées par ailleurs - si, par exemple, les explications doivent inclure à la fois des facteurs objectifs ou structurels et des facteurs subjectifs (comme l'irresponsabilité ou l'erreur), et établir 
des liens cohérents entre les divers aspects de la crise (l'échec des banques et la dette publique), plutôt que d'en souligner certains et d'en ignorer d'autres. L'examen critique des arguments, de la manière dont ils représentent et expliquent les circonstances, pourra donner naissance à un processus de connaissance, à un questionnement général de ce qui a été communément considéré comme « explication » dans le débat public : il pourra en offrir de nouvelles qui seront, telle est notre hypothèse, très proches d'explications susceptibles d'être acceptées par l'analyse sociale critique.

Nous insistons ici sur le potentiel infini du débat délibératif comme genre. Nous avons dit plus haut que la délibération pouvait être instrumentale, limitée au débat sur les moyens alternatifs de réaliser des objectifs donnés, ou bien non instrumentale, incluant la délibération sur les buts, les valeurs et les circonstances. Nous avons également remarqué précédemment que d'autres questions critiques pouvaient être ajoutées à la liste suggérée par Walton, mais nous supposons en fait qu'il n'existe pas de liste de questions définitive, et que de nouvelles interrogations peuvent en principe toujours être ajoutées. Alors que la délibération concrète présente des limites particulières, comme par exemple le fait de se limiter à la délibération instrumentale, l'un des aspects du potentiel cognitif de la délibération est autodirigé : on peut apprendre à transcender certaines limites de la délibération dans diverses directions. En termes concrets, c'est la libération du potentiel communicatif et critique de la rationalité discuté par Habermas. Ce que nous avons brièvement mentionné ici, c'est la manière dont notre compréhension de l'argumentation et du débat délibératif pratiques améliore la capacité de notre modèle de CDA à étendre au discours, d'une manière analytique concrète, la perspective de la «dialectique des Lumières » développée par Habermas.

51 Nous pensons que la version de la CDA que nous développons (dans Fairclough \& Fairclough 2012), qui incorpore notre vue spécifique de l'argumentation et du débat délibératif pratiques, est particulièrement en mesure ${ }^{7}$ de faire avancer les buts de la théorie et de la science sociale critiques, du moins telles qu'elles sont envisagées par Habermas $^{8}$, et pour promouvoir les objectifs à long terme de notre modèle de CDA : étendre la critique au discours. Elle peut aider à résoudre la préoccupation de la critique explicative de montrer comment des stratégies particulières et les imaginaires qui y sont associés tendent à prévaloir sur les autres, ainsi dans le cas des réponses politiques à la crise actuelle, à travers une analyse du débat public qui examine comment les raisons particulières pour agir que fournissent les discours peuvent être acceptées comme support rationnel des actions, et en suggérant pourquoi elles peuvent résister à un questionnement critique justifié, en partie en raison des limites du potentiel critique de délibération. Ce faisant, elle peut fournir des modèles pour transcender ces limites qui, lorsque les conditions s'y prêtent, sont susceptibles de contribuer à rendre la délibération plus approfondie et plus efficace, à remettre en question des stratégies gagnantes mais viciées (et à révéler leurs aspects manipulateurs et idéologiques), à faciliter le processus de connaissance par un questionnement critique, et aider par là à la création d'un horizon de sens du débat délibératif qui permette de produire des imaginaires et des stratégies alternatifs, qui, sous certaines conditions, sont susceptibles de contribuer à produire des états sociaux plus justes, plus équitables, plus honnêtes et plus sûrs que ceux qui prévalent actuellement. Nous n'avons cependant pas l'illusion qu'un processus amélioré du débat délibératif soit une 
condition suffisante pour obtenir de tels résultats, qui dépendent de facteurs tant matériels que sémiotiques, et des relations dialectiques entre eux.

\section{BIBLIOGRAPHIE}

Audi, Robert. 2006. Practical Reasoning and Ethical Decision (London : Routledge)

Chouliaraki, Lilie \& Norman Fairclough. 1999. Discourse in Late Modernity (Edinburgh : Edinburgh University Press)

Eemeren, Franz van. 2010. Strategic Manoeuvring in Argumentative Discourse (Amsterdam : John Benjamin's)

Eemeren, Franz von \& Robert Grootendorst. 1992. Argumentation, Communication and Fallacies (Hillsdale, NJ : Lawrence Erlbaum Associates)

Eemeren, Franz von \& Robert Grootendorst. 2004. A Systematic Theory of Argumentation. The pragma-dialectical approach (Cambridge : Cambridge University Press)

Eemeren, Franz van, Bart Grassen \& Bert Meuffels. 2009. Fallacies and Judgements of Reasonableness. Empirical Research Concerning the Pragma-Dialectical Discussion Rules (Dordrecht : Springer)

Elliott, Larry. 2010. « Labour needs to admit what it got wrong », The Guardian, 3 May 2010

Elliott, Larry \& al. 2011. « Britain's shrinking economy: panel verdict », The Guardian 25 January, 2011

Fairclough, Isabella \& Norman Fairclough. 2011. « Practical reasoning in political discourse: the UK government's response to the economic crisis in the 2008 Pre-Budget Report ", Discourse \& Society 22(3), 243-268

Fairclough, Isabella \& Norman Fairclough. 2012. Political Discourse Analysis. A Method for Advanced Students (London : Routledge)

Fairclough, Norman. 1989. Language and Power (London : Longman) ( $2^{\mathrm{e}}$ éd. 2001)

Fairclough, Norman. 1992. Discourse and Social Change (Cambridge : Polity Press)

Fairclough, Norman. 2001. « The dialectics of discourse », Textus 14, 231-242

Fairclough, Norman. 2000a. New Labour, New Language? (London : Routledge)

Fairclough, Norman. 2000b. « Dialogue in the public sphere », Sarangi, Srikant \& Malcolm Coulthard (éds.) Discourse and Social Life (London : Longman)

Fairclough, Norman. 2003. Analysing Discourse. Textual Analysis for Social Research (London : Routledge)

Fairclough, Norman. 2004/2011. "Semiotic aspects of social transformation and learning", Rogers, Rebecca (ed.). Critical Discourse Analysis in Education (New York and London: Routledge, $2^{\mathrm{e}}$ éd. 2011), 119-127

Fairclough, Norman. 2006. Language and Globalisation (London : Routledge) 
Fairclough, Norman. 2010. Critical Discourse Analysis. The Critical Study of Language, $2^{\mathrm{e}}$ éd. (London : Longman)

Fairclough, Norman \& Phil Graham. 2002. «Marx as critical discourse analyst : the genesis of a critical method and its relevance to the critique of global capital », Estudios de Sociolinguistica 3(1), 185-229, et Fairclough 2010, 301-346

Fairclough, Norman, Bob Jessop \& Andrew Sayer. 2004. « Critical realism and Semiosis », Joseph, John \& John Roberts (eds.) Realism, Discourse and Deconstruction (London : Routledge), 23-42

Forchtner, Bernhard. 2011. «Critique, the discourse-historical approach, and the Frankfurt School », Critical Discourse Studies 8(1), 1-14

Habermas, Jürgen. 1984. The Theory of Communicative Action. Volume One : Reason and the Rationalization of Society, trans. T. McCarthy (London: Heinemann) [Trad. fr. : 1987. Théorie de l'agir communicationnel. Volume Un : Rationalité de l'agir et rationalisation de la société (Paris : Fayard)]

Habermas, Jürgen. 1987. The Philosophical Discourse of Modernity, trans. Frederick Lawrence (Cambridge : Polity Press) [Trad. fr.: 2011 [1988]. Le discours philosophique de la modernité (Paris : Gallimard)]

HM Treasury. 2010. « Budget statement by the Chancellor of the Exchequer, the Rt Hon George Osborne MP », 22 Juin 2010, en ligne : http://www.hm-treasury.gov.uk/junebudget_speech.htm (conulsté le 25 Juin 2010)

Ives, Peter. 2004. Gramsci's Politics of Language. Engaging the Bakhtin Circle and the Frankfurt School (Toronto, Buffalo \& London : University of Toronto Press)

Jessop, Bob. 2004. «Critical semiotic analysis and cultural political economy », Critical Discourse Studies 1(2), 159-174

Jessop, Bob. 2008. State Power (Cambridge: Polity Press)

Jessop, Bob \& Ngai-Ling Sum. 2001. « Pre-disciplinary and post-disciplinary perspectives in political economy », New Political Economy 6, 89-101

Kompridis, Nikolas. 2006. Critique and Disclosure (Cambridge, MA \& London : MIT Press)

Marsden, Richard. 1999. The Nature of Capitalism : Marx after Foucault (London : Routledge)

Meacher, Michael. 2010. " The rich really are now filthy rich thanks to Mandelson », Left Futures (weblog), 27 janvier, en ligne : http://www.michaelmeacher.info/weblog/2010/01/the-richreally-are-now-filthy-rich-thanks-to-mandelson/ (consulté le 15 Août 2010).

Milne, Seamus. 2010. «Budget 2010 : Osborne's claims of fairness are now exposed as a fraud », The Guardian, 23 juin 2010.

Nussbaum, Martha. 2000. Women and Human Development : The Capabilities Approach (Cambridge : Cambridge University Press)

Sayer, Andrew. 2011. Why Things Matter to People : Social Science, Values and Ethical Life (Cambridge : Cambridge University Press)

Trichet, Jean-Claude. 2010. « Stimulate no more - it is now time for all to tighten », Financial Times, 22 juillet 2010.

Walton, Douglas. 2006. Fundamentals of Critical Argumentation (New York : Cambridge University Press) 
Walton, Douglas. 2007. « Evaluating practical reasoning », Syntheses 157, 197-240

Wolf, Martin. 2010. « Why the Balls critique is correct », Financial Times, 2 septembre 2010

\section{NOTES}

1. Notons que, dans cet article, nous utilisons le terme "dialectique » dans deux acceptions différentes. Nous avons discuté ci-dessus la nature dialectique des relations entre le discours et les autres éléments sociaux. Nous utilisons le terme ici en relation avec la « dialectique » comme un aspect ou une perspective sur l'argumentation, par opposition à la «logique» ou la « rhétorique ».

2. Trésor de Sa Majesté: département exécutifdugouvernement britanniquechargé de l'élaboration et de la mise en place desfinances publiqueset despolitiques économiques.

3. Pour une reconstruction plus détaillée, voir Fairclough \& Fairclough (2012). Une reconstruction complète de l'argumentation du discours tout entier est parfaitement possible en principe, bien que celle-ci soit extrêmement complexe (elle comprend par exemple des arguments distincts pour les divers éléments de la stratégie, tels que la réforme du système d'assistance sociale, et ses applications dans les divers domaines d'assistance).

4. Nous faisons référence au premier des deux sens du terme «dialectique " que nous avons distingué dans la note 1 .

5. D'après notre propre expérience, cette perspective non dialectique est répandue même parmi les spécialistes de l'analyse critique du discours. Par exemple, une des réponses que nous rencontrons souvent à notre proposition de donner la primauté à l'analyse de l'argumentation dans l'analyse du discours politique revient souvent à l'affirmation du courant non dialectique selon laquelle la raison n'est que le masque du pouvoir (Fairclough \& Fairclough 2012 : 13-15).

6. Notons le lien étroit impliqué ici entre l'action communicative et le raisonnement pratique.

7. C'est peut-être en fait la seule qui le soit. Aucune autre version de la CDA ne possède le caractère dialectique auquel nous faisons appel, ni ne fournit de traitement satisfaisant de l'argumentation en général ou de l'argumentation pratique et du débat délibératif en particulier. Nous trouvons utile la description que donne Forchtner (2011) de l'Ecole de Francfort, mais ne comprenons pas pourquoi il considère «l'approche discursive- historique " comme particulièrement bien placée pour promouvoir les préoccupations et les objectifs d'Habermas dans le cadre de la $\mathrm{CDA}$, car à notre avis, elle ne remplit pas certaines conditions préalables cruciales à cet égard.

8. Nous réservons pour une autre occasion la manière dont notre approche de la CDA et de l'argumentation (pratique) appréhende les débats au sein de la théorie critique et du marxisme sur la relation entre la critique et la raison. Ives (2004) par exemple se prononce en faveur d'une position gramscienne sur ce problème contre Habermas, et Kompridis (2006) préconise une alternative heideggérienne à Habermas. Notre intention n'est pas d'adopter une position habermassienne, mais d'effectuer une première approche de ces débats en discutant les théories d'Habermas, dont l'approche des questions de la communication et du discours est d'une qualité, d'un niveau et d'une complexité telles qu'elles en font un point de départ évident. 


\section{RÉSUMÉS}

Cet article représente le développement de notre récent travail sur le discours politique, centré sur les réactions politiques à l'actuelle crise financière et économique en Grande-Bretagne. Considérant le discours politique comme doté d'un caractère essentiellement argumentatif, et plus spécifiquement comme une forme d'argumentation et de délibération pratiques, nous soutenons qu'une analyse critique de ce discours appelle à intégrer la théorie et l'analyse de l'argumentation à la version de l'analyse critique du discours (CDA) avec laquelle nous travaillons. La présentation de cette version de la CDA, et de notre approche de l'analyse et de l'évaluation de l'argumentation et de la délibération pratiques, est suivie d'une illustration de la démarche. Celle-ci se réfère à une partie du discours sur le Budget prononcé en juin 2010 par Georges Osborne, Chancelier de l'Echiquier du gouvernement britannique de coalition entre conservateurs et libéraux démocrates, et à la mise en cause critique de la stratégie d'austérité du gouvernement qui eu lieu dans le débat public, particulièrement dans les média. Dans la dernière partie de cet article, nous avançons que notre approche innovatrice de l'analyse et de l'évaluation de l'argumentation et de la délibération pratiques contribue de manière particulièrement efficace à promouvoir l'objectif de la CDA : étendre au discours les formes de critique répandues dans le champ de la théorie sociale critique. Nous nous appuyons pour ce faire sur les débats qui traitent des rapports entre critique et rationalité. Nous posons que l'approche normative que nous adoptons est nécessaire pour intégrer l'analyse du discours argumentatif à la critique, et que notre démarche est en parfait accord avec les objectifs de la théorie critique, du moins tels que Habermas les conçoit.

This paper is a development of our recent work on political discourse, which has focused upon British political responses to the current financial and economic crisis. We take political discourse to be primarily argumentative in character, and more specifically to be primarily practical argumentation and deliberation, and we argue that critical analysis of political discourse requires us to incorporate argumentation theory and analysis into the version of critical discourse analysis (CDA) which we work with. We present this version of CDA, and our approach to analysis and evaluation of practical argumentation and deliberation, and then illustrate our approach with reference to part of the Budget speech which George Osborne, Chancellor of the Exchequer in the British Conservative-Liberal Democratic Coalition government, gave in June 2010 and to the critical questioning of the government's austerity strategy in public deliberation especially in the media. In the final section of the paper, we argue that our innovative approach to analysing and evaluating practical argumentation and deliberation is particularly effective in pursuing CDA's objective to extend forms of critique familiar in critical social theory to discourse, drawing upon debates over the relationship of critique to rationality. We argue that the normative approach that we adopt is necessary for integrating analysis of argumentative discourse into critique, and that our approach is strongly consistent with the objectives of critical theory, at least as Habermas conceives them.

\section{INDEX}

Keywords : critique and rationality, critique of discourse, deliberation, financial and economical crisis, Habermas (Jürgen), political discourse, practical argumentation

Mots-clés : argumentation pratique, crise financière et économique, critique du discours, critique et rationalité, délibération, discours politique, Habermas (Jürgen) 


\section{AUTEURS}

\section{ISABELA FAIRCLOUGH}

Université du Lancashire Central, GB

NORMAN FAIRCLOUGH

Université de Lancaster, GB 